\title{
Hacking Blind Navigation
}

\section{João Guerreiro \\ Carnegie Mellon University \\ jpvguerreiro@cmu.edu \\ Jeffrey P. Bigham \\ Carnegie Mellon University \\ jbigham@cs.cmu.edu}

\section{Daisuke Sato \\ IBM Research - Tokyo \\ dsato@jp.ibm.com}

\author{
Hernisa Kacorri \\ University of Maryland, College Park \\ hernisa@umd.edu
}

\author{
Edward Cutrell \\ Microsoft Research - Redmond \\ cutrell@microsoft.com
}

\author{
Dragan Ahmetovic \\ University of Turin \\ dragan.ahmetovic@unito.it
}

\section{Chieko Asakawa}

Carnegie Mellon University \& IBM Research

chiekoa@cs.cmu.edu

\section{ABSTRACT}

Independent navigation in unfamiliar and complex environments is a major challenge for blind people. This challenge motivates a multi-disciplinary effort in the $\mathrm{CHI}$ community aimed at developing assistive technologies to support the orientation and mobility of blind people, including related disciplines such as accessible computing, cognitive sciences, computer vision, and ubiquitous computing. This workshop intends to bring these communities together to increase awareness on recent advances in blind navigation assistive technologies, benefit from diverse perspectives and expertises, discuss open research challenges, and explore avenues for multi-disciplinary collaborations. Interactions

Permission to make digital or hard copies of part or all of this work for personal or classroom use is granted without fee provided that copies are not made or distributed for profit or commercial advantage and that copies bear this notice and the full citation on the first page. Copyrights for third-party components of this work must be honored. For all other uses, contact the owner/author(s)

CHI'19 Extended Abstracts, May 4-9, 2019, Glasgow, Scotland UK

(c) 2019 Copyright held by the owner/author(s).

ACM ISBN 978-1-4503-5971-9/19/05.

https://doi.org/10.1145/3290607.3299015 
are fostered through a panel on Open Challenges and Avenues for Interdisciplinary Collaboration, Minute-Madness presentations, and a Hands-On Session where workshop participants can hack (design or prototype) new solutions to tackle open research challenges. An expected outcome is the emergence of new collaborations and research directions that can result in novel assistive technologies to support independent blind navigation.

\section{CCS CONCEPTS}

- Human-centered computing $\rightarrow$ Accessibility technologies.

\section{KEYWORDS}

Accessibility; orientation and mobility; blind navigation; assistive technologies; visual impairment.

\section{ACM Reference Format:}

João Guerreiro, Hernisa Kacorri, Jeffrey P. Bigham, Edward Cutrell, Daisuke Sato, Dragan Ahmetovic, and Chieko Asakawa. 2019. Hacking Blind Navigation. In CHI Conference on Human Factors in Computing Systems Extended Abstracts (CHI'19 Extended Abstracts), May 4-9, 2019, Glasgow, Scotland UK. ACM, New York, NY, USA, 8 pages. https://doi.org/10.1145/3290607.3299015

\section{BACKGROUND}

Orientation and Mobility (O\&M) skills are essential for the independence of people who are blind. O\&M training, exploration of tactile maps and navigation aids such as the white cane and guide dogs help blind people to navigate and acquire knowledge about their surroundings. However, this knowledge is often insufficient, as most blind people avoid to visit unfamiliar places by themselves and can a have fragmented or incomplete understanding of familiar environments [9, 26].

There is a multidisciplinary effort and a great amount of research trying to understand and support the $O \& M$ of blind people in areas such as human-computer interaction, accessible computing, cognitive sciences (e.g. psychology, neuroscience), computer vision, and ubiquitous computing, among others. These efforts created a variety of solutions that help blind people navigate in the real-world and acquire spatial knowledge either directly or indirectly (e.g. by exploring maps or virtual environments)

Solutions that provide in-situ support to blind travelers include turn-by-turn navigation to help users reach a destination. Several researchers have been tackling this challenge with different approaches and from different perspectives. This includes, for instance, efforts to assess the requirements and information needs for blind navigation [2, 21]; the design and evaluation of interfaces to guide the user $[6,19,24]$; the study or modeling of user behavior during navigation assistance [13, 15, 22]; the investigation of the factors that influence user acceptance of such systems [1]; and the use of crowdsourcing to ease the installation and maintenance of indoor localization infrastructures [10] Still, most solutions present static interfaces that are not able to adapt to users nor different situations. 


\section{Sidebar 1: Workshop Objectives}

Increase awareness of recent advances across different but related fields. We believe that such awareness, together with the different perspectives and expertise of different communities will result in insightful discussions and future collaborations.

Surface open challenges in this area with invited researchers through an interactive panel on Open Challenges and Avenues for Interdisciplinary Collaboration. The challenges addressed in the workshop will be based on participants' position papers and a list of potential challenges collected by the organizers. These challenges include: sharing large-scale data to build new knowledge; easing the transfer from research efforts to real-world deployment; and further exploring non-visual augmented and virtual reality to help gaining knowledge of the environment.

Design and prototype new solutions with a Hands-On, hacking session to tackle open challenges. This aims to be a starting point for multidisciplinary collaborations and novel research directions that can further advance the stateof-the-art on assistive technologies to support independent navigation.

Share resources and data. Create awareness of currently available datasets and encourage the increase of resources and data sharing among researchers and communities while considering privacy.

Establish metrics and methods for benchmarking progress that allow for comparison across different systems and user studies.
Potential avenues for future research may arise from the increasing effort to release large-scale usage datasets $[8,14]$. The availability of such datasets can promote new data-driven approaches to study and model user behavior, improve interface design, and support adaptive interfaces.

Besides turn-by-turn guidance, several commercial and research solutions try to convey more information about the user's surroundings. For instance, Footnotes [11] is built on top of Microsoft's Soundscape and provides rich textual descriptions containing functional, visual, historical and social descriptions. Future research can further explore how to maximize blind users' knowledge of the surroundings (with reduced cognitive load) with non-visual augmented reality.

An area of increasing relevance for the $O \& M$ of blind people is computer vision, which has been used to help guiding or identifying elements of interest in the environment, such as crosswalks or particular objects/obstacles [4, 7, 20,27], sometimes combined with crowdsourcing [3]. While a powerful tool already, real-time usage of computer vision models in mobile or wearable devices is still a challenge.

In addition to navigation skills, O\&M classes teach blind people how to explore tactile maps to gain prior knowledge of the environment. Recent research has investigated how to make those maps interactive in order to overcome limitations of traditional tactile maps (e.g. learning braille) [5].

An alternative way to explore the environment beforehand is through virtual navigation, for instance by using a combination of audio-haptic feedback or 3D audio [12, 18, 23, 28]. While efforts to make virtual environments more immersive are needed, it is also crucial to ease the creation of real-world representations so that these solutions can be more easily made available to the end users.

Cognitive sciences play a very important role in the design and evaluation of blind navigation solutions. Literature about spatial knowledge acquisition and how blind users build a mental representation of an environment is the scaffold for the design of many technological solutions [25]. To cite one example, solutions that use 3D audio rely on findings that spatial audio outperforms spatial language in cognitively demanding scenarios [17]. Also, these disciplines are also essential to assess the effectiveness of new solutions [16].

\section{OBJECTIVES}

While the aforementioned contributions are just a subset of recent research supporting independent navigation for blind people, these contributions are often spread out in different research communities that do not often collaborate. However, some of the challenges can only be tackled if researchers from different fields are aware of each others' progress and work together to leverage the skills of each community. For instance, computer vision is key to detect and avoid obstacles while navigating, but interface design expertise is needed to understand how to convey that information appropriately. This workshop intends to connect researchers working on similar and complementary areas related to blind navigation in order to achieve the goals outlined in Sidebar 1. 


\section{ORGANIZERS}

The organizers are experts in disciplines relevant to blind navigation, including $\mathrm{HCl}$, accessible computing, cognitive sciences, AI, and machine learning.

João Guerreiro is a Project Scientist at Carnegie Mellon University. His research focuses on supporting independent navigation of people with visual impairments using either real-world navigation assistance or virtual navigation to gain prior knowledge of the environment. He obtained a PhD from the University of Lisbon on the use of non-visual interfaces with simultaneous speech to speed-up blind people's access to personally relevant digital information. João Guerreiro is the main contact person for this workshop.

Hernisa Kacorri is an Assistant Professor at University of Maryland, College Park. Her expertise is on technologies that leverage artificial intelligence to address human challenges, faced due to health or disability. Her work emphasises rigorous experimental methodologies and early user involvement to assess impact. Her recent work on technologies for people with visual impairments focuses on personalizing object recognizers through teachable machines, as well as uncovering behavioral patterns and environmental factors from real-world mobility data.

Jeffrey P. Bigham is an Associate Professor and PhD Director in the Human-Computer Interaction and Language Technologies Institutes in the School of Computer Science at Carnegie Mellon University. Dr. Bigham's research combines crowdsourcing and machine learning to make novel deployable interactive systems, and ultimately solve hard problems in computer science. Many of these systems are designed with a deep understanding of the needs of people with disabilities to be useful in their everyday lives. He received his B.S.E degree in Computer Science from Princeton University in 2003, and received his Ph.D. in Computer Science and Engineering from the University of Washington in 2009. He has received the Alfred P. Sloan Foundation Fellowship, the MIT Technology Review Top 35 Innovators Under 35 Award, and the National Science Foundation CAREER Award.

Edward Cutrell is a Principal Researcher at Microsoft Research where he explores computing for disability, accessibility, and inclusive design in the Ability Group. Over the years, he has worked on a broad range of $\mathrm{HCl}$ topics and technologies including input technologies, search interfaces, intelligent notifications, and systems useful for people living in underserved rural and urban communities in developing countries. He also holds an affiliate faculty appointment in the Information School at the University of Washington. Ed has worked in the field of $\mathrm{HCl}$ since 2000 and received his PhD from the University of Oregon in cognitive neuropsychology.

Daisuke Sato is a Technical Leader in the Accessibility Research Group at IBM Research - Tokyo. $\mathrm{He}$ is leading the development of cognitive assistance research to help visually impaired people regain information in the real world. He is the main contributor of the NavCog project, a smartphone-based indoor navigation app for visually impaired people with accurate and scalable positioning. He obtained 
a PhD and a MSc from the University of Tsukuba, Japan. His PhD focused on interfaces to improve accessibility through cooperative efforts by visually impaired people and remote workers.

Dragan Ahmetovic is a Postdoctoral Fellow at the University of Turin, Italy. His research focuses on improving the quality of life of individuals with disabilities by overcoming barriers that limit their access to information and the physical world. He works on assistive technologies that augment cognitive capabilities of the users, through embodied sensing, knowledge retrieval, and human computer interaction, mediated by mobile and pervasive devices, during tasks such as mobility, surroundings awareness and information access.

Chieko Asakawa is an IBM Fellow since 2009. Series of pioneering technologies generated under Chieko's leadership at IBM Research Tokyo significantly contributed in advancing information accessibility in the last three decades. Today, Chieko is focusing on advancing cognitive assistant research to help the blind regain information by augmenting missing or weakened abilities in the real world. She was inducted into the Women in Technology International (WITI) Hall of Fame in 2003. In 2013, the government of Japan awarded her the Medal of Honor with Purple Ribbon. She has been also serving as an IBM Distinguished Service Professor at Carnegie Mellon University.

\section{WEBSITE}

We will use our website at https://blindnavigationCHI19.wordpress.com to publicize the workshop and provide access to its content. This includes publishing the call for participation and submission details, the accepted position papers, and the ability to start the discussion prior to the workshop.

\section{PRE-WORKSHOP PLANS}

The call for participation will be broadly distributed to different research communities addressing the subject of the workshop. This includes posting to mailing lists (e.g., chi-announcements) and to social media groups (e.g., ASSETS conference and SigCHI-Access), and using the organizers' personal networks to contact researchers with relevant contributions in the area.

We will ask for position papers describing the authors' past and current research in this area. We encourage authors to include at least one research challenge or scenario that they would like to tackle during the workshop. Position papers should have a maximum of four pages in the SIGCHI Extended Abstract format. Their acceptance is based on relevance for the workshop theme, diversity of research communities and the potential impact and novelty of the authors' research.

All accepted position papers will be made available on the workshop website. We will encourage participants to read each others papers and to start the discussion prior to the workshop. We intend to involve blind people through the social media and our personal networks, and encourage them to submit questions for our interactive panel. 
Table 1: Workshop Schedule.

\begin{tabular}{|l|l|}
\hline $09: 00$ & A Welcome Note by the Organizers \\
\hline $09: 15$ & $\begin{array}{l}\text { Interactive Panel: } \\
\text { Open Challenges and Avenues } \\
\text { for Interdisciplinary Collaboration }\end{array}$ \\
\hline $10: 45$ & Coffee Break \\
\hline $11: 00$ & Minute Madness Presentations \\
\hline $12: 00$ & $\begin{array}{l}\text { Outline Research Challenges } \\
\text { and Group Formation }\end{array}$ \\
\hline $12: 30$ & Lunch \\
\hline $13: 30$ & $\begin{array}{l}\text { Hacking Blind Navigation: } \\
\text { Hands-On Session }\end{array}$ \\
\hline $15: 30$ & Coffee Break \\
\hline $15: 45$ & $\begin{array}{l}\text { Hacking Blind Navigation: } \\
\text { Hands-On Session }\end{array}$ \\
\hline $16: 45$ & Group Summary Presentations \\
\hline $17: 00$ & Wrap-Up and Follow-Up Plans \\
\hline
\end{tabular}

\section{WORKSHOP STRUCTURE}

We propose a one-day workshop with 20 participants. We envision a highly interactive workshop with the schedule outlined in Table 1. The workshop structure consists of an Interactive Panel focused on Open Challenges and Avenues for Interdisciplinary Collaboration that aims to foster the discussion among different communities and try to establish a common ground for future collaborations in the theme of the workshop. Then, Minute Madness presentations aim to briefly introduce the participants and their research. Afterwards, participants will be asked to form groups to design or prototype new solutions, based on the challenges presented in their position papers.

\section{POST-WORKSHOP PLANS}

We expect this workshop to influence future research in this area. It is our goal that both the discussion in the interactive panel and the hands-on session will benefit from the participants' different perspectives and expertise. Each group will be encouraged to present the main outcomes of their new designs and prototypes. In addition, the organizers will write a report summarizing the main outcomes of this workshop to either ACM Interactions or ACM SIGACCESS Newsletter. Finally, we will reflect on the lessons learned from the workshop, by summarizing its key take-away messages and planning for next steps.

\section{CALL FOR PARTICIPATION}

Independent navigation is a major challenge for people with visual impairments, in particular in unfamiliar or complex environments. There is a multi-disciplinary effort in the $\mathrm{CHI}$ community to support independent navigation through assistive technologies, but also in related disciplines, such as accessible computing, cognitive sciences and computer vision.

The aim of this workshop is to bring together these communities to increase awareness on recent advances on navigation assistive technologies. We see the field benefiting from different perspectives and expertise, discussions on open research challenges, as well as exploration of new avenues for multidisciplinary collaborations. The workshop consists of an interactive panel, minute-madness presentations and a hands-on session to design or prototype new solutions to tackle open research challenges. More information on the workshop can be found at https://blindnavigationCHI19.wordpress.com.

\section{Submissions:}

We invite researchers to submit position papers in the SIGCHI Extended Abstract format (four-page maximum, excluding references). Papers should summarize authors' past and current research in the workshop theme, and one open research challenge for discussion in the workshop. Submissions should be sent to blindnavigationCHI19@gmail.com in PDF format. Position papers will be peer-reviewed based on relevance to the workshop theme, diversity of research communities, and the potential for 
impact and novelty. At least one co-author of each accepted paper must attend the workshop and all participants must register for the workshop and for at least one day of the conference.

\section{Important Dates:}

- Submission Deadline: 12 February 2019

- Notification: 1 March 2019

- Workshop: 4 May 2019

\section{ACKNOWLEDGMENTS}

We would like to thank ACM SIGACCESS for sponsoring and supporting this workshop.

\section{REFERENCES}

[1] Ali Abdolrahmani, William Easley, Michele Williams, Stacy Branham, and Amy Hurst. 2017. Embracing Errors: Examining How Context of Use Impacts Blind Individuals' Acceptance of Navigation Aid Errors. In Proceedings of the $2017 \mathrm{CH}$ Conference on Human Factors in Computing Systems (CHI '17). ACM, 4158-4169.

[2] Nikola Banovic, Rachel L Franz, Khai N Truong, Jennifer Mankoff, and Anind K Dey. 2013. Uncovering information needs for independent spatial learning for users who are visually impaired. In Proceedings of the 15th International ACM SIGACCESS Conference on Computers and Accessibility. ACM, 24.

[3] Jeffrey P Bigham, Chandrika Jayant, Andrew Miller, Brandyn White, and Tom Yeh. 2010. VizWiz:: Locatelt-enabling blind people to locate objects in their environment. In Computer Vision and Pattern Recognition Workshops (CVPRW), 2010 IEEE Computer Society Conference on. IEEE, 65-72.

[4] James Coughlan and Roberto Manduchi. 2009. Functional assessment of a camera phone-based wayfinding system operated by blind and visually impaired users. International Journal on Artificial Intelligence Tools 18, 03 (2009), 379-397.

[5] Julie Ducasse, Anke M Brock, and Christophe Jouffrais. 2018. Accessible interactive maps for visually impaired users. In Mobility of Visually Impaired People. Springer, 537-584.

[6] Navid Fallah, Ilias Apostolopoulos, Kostas Bekris, and Eelke Folmer. 2013. Indoor human navigation systems: A survey. Interacting with Computers 25, 1 (2013), 21-33.

[7] Vítor Filipe, Filipe Fernandes, Hugo Fernandes, António Sousa, Hugo Paredes, and João Barroso. 2012. Blind navigation support system based on Microsoft Kinect. Procedia Computer Science 14 (2012), 94-101.

[8] German H Flores and Roberto Manduchi. 2018. WeAllWalk: An Annotated Dataset of Inertial Sensor Time Series from Blind Walkers. ACM Transactions on Accessible Computing (TACCESS) 11, 1 (2018), 4.

[9] Nicholas A. Giudice and Gordon E. Legge. 2008. Blind Navigation and the Role of Technology. John Wiley \& Sons, Inc.

[10] Cole Gleason, Dragan Ahmetovic, Saiph Savage, Carlos Toxtli, Carl Posthuma, Chieko Asakawa, Kris M. Kitani, and Jeffrey P. Bigham. 2018. Crowdsourcing the Installation and Maintenance of Indoor Localization Infrastructure to Support Blind Navigation. Proc. ACM Interact. Mob. Wearable Ubiquitous Technol. 2, 1, Article 9 (March 2018), 25 pages.

[11] Cole Gleason, Alexander J Fiannaca, Melanie Kneisel, Edward Cutrell, and Meredith Ringel Morris. 2018. FootNotes: Geo-referenced Audio Annotations for Nonvisual Exploration. Proceedings of the ACM on Interactive, Mobile, Wearable and Ubiquitous Technologies 2, 3 (2018), 109.

[12] João Guerreiro, Dragan Ahmetovic, Kris M Kitani, and Chieko Asakawa. 2017. Virtual Navigation for Blind People: Building Sequential Representations of the real-world. In The proceedings of the 19th international ACM SIGACCESS conference on Computers and accessibility. ACM. 
[13] João Guerreiro, Eshed Ohn-Bar, Dragan Ahmetovic, Kris Kitani, and Chieko Asakawa. 2018. How Context and User Behavior Affect Indoor Navigation Assistance for Blind People. In Proceedings of the Internet of Accessible Things (W4A '18). ACM, Article 2, 2:1-2:4 pages.

[14] Hernisa Kacorri, Sergio Mascetti, Andrea Gerino, Dragan Ahmetovic, Hironobu Takagi, and Chieko Asakawa. 2016. Supporting Orientation of People with Visual Impairment: Analysis of Large Scale Usage Data. In International ACM SIGACCESS Conference on Computers and Accessibility. ACM.

[15] Hernisa Kacorri, Eshed Ohn-Bar, Kris M. Kitani, and Chieko Asakawa. 2018. Environmental Factors in Indoor Navigation Based on Real-World Trajectories of Blind Users. In Proceedings of the 2018 CHI Conference on Human Factors in Computing Systems (CHI '18). ACM, Article 56, 56:1-56:12 pages.

[16] Rob Kitchin and RD Jacobson. 1997. Techniques to collect and analyze the cognitive map knowledge of persons with visual impairment or blindness: issues of validity. Journal of Visual Impairment and Blindness 91, 4 (1997), 360-376.

[17] Roberta L Klatzky, James R Marston, Nicholas A Giudice, Reginald G Golledge, and Jack M Loomis. 2006. Cognitive load of navigating without vision when guided by virtual sound versus spatial language. Journal of Experimental Psychology: Applied 12, 4 (2006), 223.

[18] Orly Lahav and David Mioduser. 2008. Haptic-feedback support for cognitive mapping of unknown spaces by people who are blind. International Journal of Human-Computer Studies 66, 1 (2008), 23-35.

[19] Jack M Loomis, Reginald G Golledge, and Roberta L Klatzky. 1998. Navigation system for the blind: Auditory display modes and guidance. Presence: Teleoperators and Virtual Environments 7, 2 (1998), 193-203.

[20] Sergio Mascetti, Dragan Ahmetovic, Andrea Gerino, and Cristian Bernareggi. 2016. ZebraRecognizer: Pedestrian Crossing Recognition for People with Visual Impairment or Blindness. Pattern Recognition (2016).

[21] Hugo Nicolau, Joaquim Jorge, and Tiago Guerreiro. 2009. Blobby: how to guide a blind person. In Proceedings of the CHI Conference Extended Abstracts on Human Factors in Computing Systems. ACM, 3601-3606.

[22] Eshed Ohn-Bar, João Guerreiro, Dragan Ahmetovic, Kris M. Kitani, and Chieko Asakawa. 2018. Modeling Expertise in Assistive Navigation Interfaces for Blind People. In 23rd International Conference on Intelligent User Interfaces (IUI '18).

[23] Lorenzo Picinali, Amandine Afonso, Michel Denis, and Brian FG Katz. 2014. Exploration of architectural spaces by blind people using auditory virtual reality for the construction of spatial knowledge. International Journal of Human-Computer Studies 72, 4 (2014), 393-407.

[24] Daisuke Sato, Uran Oh, Kakuya Naito, Hironobu Takagi, Kris Kitani, and Chieko Asakawa. 2017. NavCog3: An Evaluation of a Smartphone-Based Blind Indoor Navigation Assistant with Semantic Features in a Large-Scale Environment. In Proceedings of the 19th International ACM SIGACCESS Conference on Computers and Accessibility (ASSETS '17). ACM.

[25] Victor R Schinazi, Tyler Thrash, and Daniel-Robert Chebat. 2016. Spatial navigation by congenitally blind individuals Wiley Interdisciplinary Reviews: Cognitive Science 7, 1 (2016), 37-58.

[26] Michele A. Williams, Amy Hurst, and Shaun K. Kane. 2013. "Pray Before You Step out": Describing Personal and Situational Blind Navigation Behaviors. In Proceedings of the 15th International ACM SIGACCESS Conference on Computers and Accessibility (ASSETS '13). ACM, Article 28, 8 pages.

[27] Limin Zeng, Markus Simros, and Gerhard Weber. 2017. Camera-based Mobile Electronic Travel Aids Support for Cognitive Mapping of Unknown Spaces. In Proceedings of the 19th International Conference on Human-Computer Interaction with Mobile Devices and Services (MobileHCI '17). ACM, Article 8, 10 pages.

[28] Yuhang Zhao, Cynthia L Bennett, Hrvoje Benko, Edward Cutrell, Christian Holz, Meredith Ringel Morris, and Mike Sinclair. 2018. Enabling People with Visual Impairments to Navigate Virtual Reality with a Haptic and Auditory Cane Simulation. In Proceedings of the SIGCHI Conference on Human Factors in Computing Systems. ACM. 